\title{
Reducing Radiation Dermatitis Using a Film-forming Silicone Gel During Breast Radiotherapy: A Pilot Randomized-controlled Trial
}

\author{
SONGMI AHN ${ }^{1}$, KIHOON SUNG ${ }^{1}$, HYUN JU KIM ${ }^{1}$, YOUNG EUN CHOI ${ }^{1}$, \\ YOUNG KYU LEE ${ }^{1}$, JEONG SOO KIM ${ }^{2}$, SEUL KI LEE ${ }^{2}$ and JOO-YOUNG ROH ${ }^{2}$ \\ ${ }^{1}$ Department of Radiation Oncology, Gachon University Gil Medical Center, \\ Gachon University School of Medicine, Incheon, Republic of Korea; \\ ${ }^{2}$ Department of Dermatology, Gachon University Gil Medical Center, \\ Gachon University School of Medicine, Incheon, Republic of Korea
}

\begin{abstract}
Background/Aim: To evaluate whether topical use of a film-forming silicone gel (StrataXRT ${ }^{\circledR}$ ) could reduce radiation dermatitis compared to a moisturizing cream ( $X$ derm $\left.^{\circledR}\right)$ in patients receiving whole breast radiotherapy. Patients and Methods: A total of 56 patients with breast cancer were randomized to use StrataXRT or X-derm. The severity of radiation dermatitis was graded using physiological skin parameters, clinician-assessed visual rating scales and patient-reported symptoms. Changes in these parameters from baseline to 4 weeks post-radiotherapy were evaluated every two weeks. Results: Two-way repeated-measures ANOVA revealed different patterns of changes in the erythema index $(F=3.609, p=0.008)$ and melanin index $(F=3.475, p=0.015)$. The post hoc analysis demonstrated a significantly lower erythema index and melanin index in the patients allocated to the StrataXRT group. Conclusion: The use of StrataXRT can reduce radiation dermatitis with respect to objectively measured physiological skin parameters. The results of the present study will support the feasibility of conducting a larger randomized controlled trial.
\end{abstract}

Radiation dermatitis (RD) is the most common adverse side effect of radiation therapy (RT), appearing in almost every

This article is freely accessible online.

Correspondence to: KiHoon Sung, MD, Ph.D., Department of Radiation Oncology, Gachon University Gil Medical Center, 21 Namdong-daero 774beon-gil, Namdong-gu, Incheon 21565, Republic of Korea. Tel: +82 324608052, Fax: +82 324602384, e-mail: novalis@gilhospital.com

Key Words: Film-forming silicone gel, StrataXRT, radiation dermatitis, breast cancer. patient with breast cancer who receives RT (1-3). The symptoms of RD generally include erythema, edema, dryness, hair loss, hyperpigmentation, and most seriously, moist desquamation, ulcers, and skin necrosis, which can cause discomfort in patients and disruption to RT (4-6). Traditionally, RD has been evaluated and graded using visual rating scales, such as the Common Terminology Criteria for Adverse Events (CTCAE) and Radiation Therapy Oncology Group (RTOG) criteria (7, 8). An additional scoring system, the modified 10-point Catterall skin scoring profile (CSSP), has been used to better evaluate $\mathrm{RD}(9,10)$. However, due to the inherent subjectivity of these scales, a number of alternative methods have been developed to objectively quantify the changes in physiological skin parameters, such as electrochemical, reflectance spectrophotometer (erythema index (EI) and melanin index (MI)), trans-epidermal water loss (TEWL), and laser Doppler flowmetry (LDF) methods (11-15).

The management of RD, as an inevitable part of RT, is directed toward the palliation of skin symptoms (16). Several agents such as steroids, hyaluronic acid, aloe vera, sucralfate, and adrenergic vasoconstrictors have been used to prevent or reduce the severity of RD $(10,17-22)$. However, there are no clear guidelines or a consensus supporting the application of any topical agent as a standard of care for RD.

Recently, silicone-based barrier-forming products have been used for the management of RD $(23,24)$. The siliconebased film dressing provides mechanical protection from skin damage and TEWL, and may prevent moist desquamation and reduce the severity of RD. Despite these advantages, this silicone-based film dressing may have limitations, such as small bolus effects, easy detachment particularly when bathing or perspiring, and the need for frequent replacement at least twice a week. Topical applications of a silicone gel have been shown to be effective 


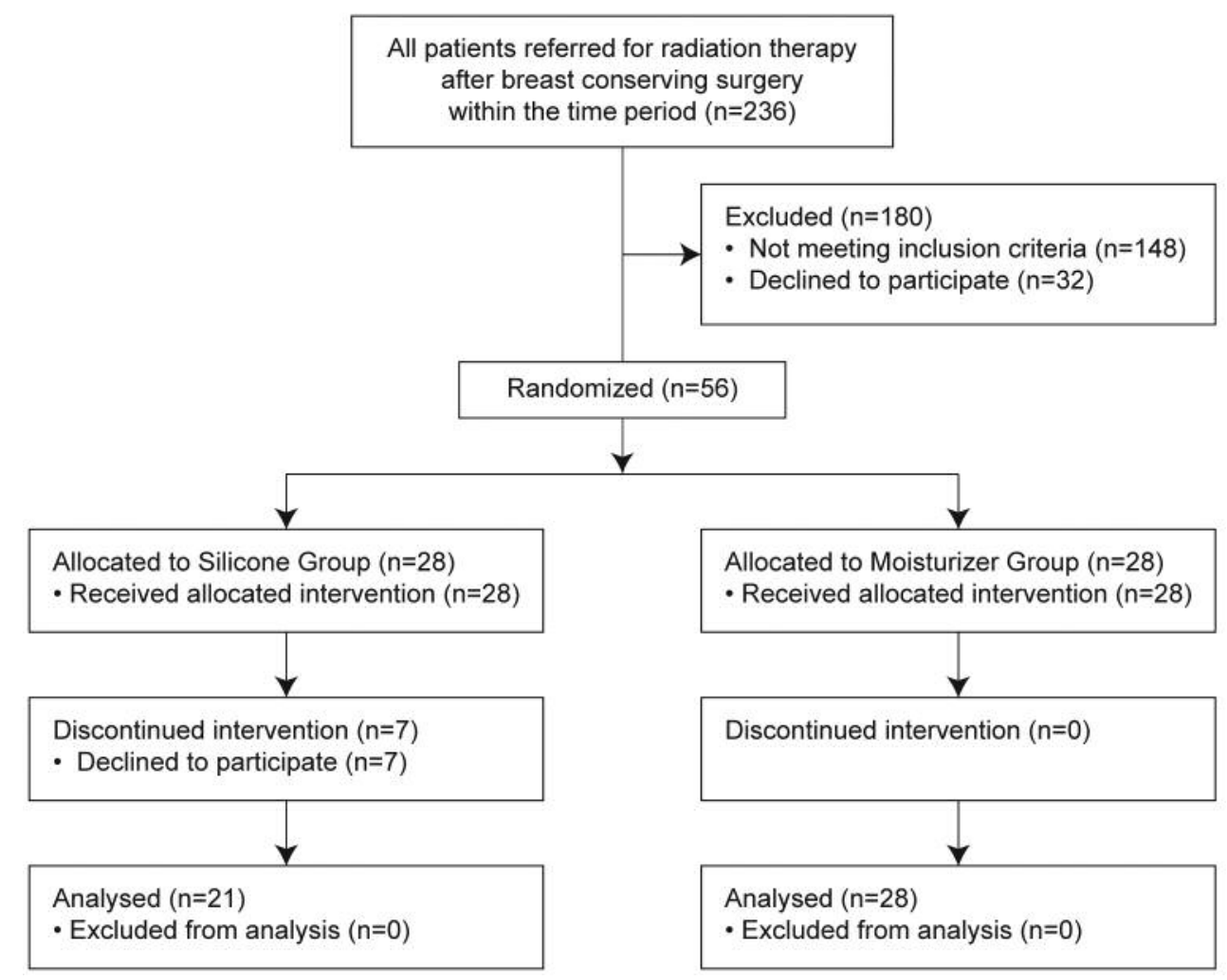

Figure 1. CONSORT flow diagram showing participant flow through each stage of the randomized controlled trial (enrolment, intervention allocation, follow-up, and data analysis).

in promoting accelerated epithelialization, reducing the inflammatory response $(25,26)$. StrataXRT ${ }^{\circledR}$ (Stratpharma AG, Basel, Switzerland) is a film-forming silicone gel designed to promote a moist wound-healing environment. When applied topicaly, StrataXRT dries to form a thin, flexible, protective layer that is gas permeable and waterproof. This environment leads to rapid wound healing and faster skin recovery. Studies have raised the possibility that topical use of StrataXRT might be effective in reducing various types of radiation-induced skin reactions $(27,28)$. A recently published randomized controlled trial has demonstrated the effectiveness of StrataXRT in preventing, delaying and reducing the severity of RD in patients with head and neck cancer (29).

The primary aim of this pilot randomized controlled trial was to compare the effectiveness of a film-forming silicone gel (StrataXRT ${ }^{\circledR}$ ) and a moisturizing cream (X-derm ${ }^{\circledR}$, Pharmbio Korea Inc., Republic of Korea) in reducing RD in patients receiving RT for breast cancer. The secondary aim was to correlate three physiological skin parameters (EI, MI, and TEWL) with changes in clinician-assessed visual rating scales (CSSP, RTOG, and CTCAE criteria) and patientreported symptoms (dryness, itchiness, burning sensation, and pain).

\section{Patients and Methods}

Trial protocol. This was a single-centre, unblinded, randomizedcontrolled parallel study that included patients with breast cancer who had been referred for adjuvant breast RT after breast conserving surgery. Female patients aged 20-60 years with stage pTis, pT1-2, pN0, M0 were recruited between May 2017 and January 2019 at Gachon University Gil Medical Center in Incheon, Republic of Korea. The exclusion criteria were previous ipsilateral breast RT, any skin disease, any skin allergy to usual topical creams, and inability to consent or comply with instructions or skin care. Participants were randomly assigned to the silicone (StrataXRT ${ }^{\circledR}$ ) versus moisturizer $\left(\mathrm{X}-\mathrm{derm}^{\circledR}\right)$ groups using a random number generator (Figure 1).

All study participants provided written informed consent with respect to the use of topical agents and clinical data management for research purposes. This trial was designed and conducted in accordance with the principles outlined in the Declaration of Helsinki and within the guidelines of Good Clinical Practices. This trial received ethics approval from the Institutional Review Board, Gachon University Gil Medical Center (IRB number: GAIRB2017127) and was retrospectively registered on Clinical Research Information Service (identifier KCT0002695).

Radiotherapy. A computed tomography (CT) simulation was performed in the supine position on a breast board with the ipsilateral arm up. The target volumes and organ-at-risk volumes 
Table I. Visual rating scales for radiation dermatitis.

\begin{tabular}{llccc}
\hline Reactions & Severity & CSSP & RTOG & CTCAE \\
\hline No reaction & & 1 & 0 & 0 \\
Erythema & Light, Tender & 2 & 1 & 1 \\
& Moderate, Bright & 3 & & \\
& Severe & 4 & & \\
Dry & $<50 \%$ & 5 & & \\
desquamation & $>50 \%$ & 6 & & \\
Blistering & Moderate & 7 & 2 & 2 \\
Edema & $<50 \%$, Patchy & 8 & & \\
Moist & $>50 \%$, Confluent, & 9 & & \\
desquamation & Skin fold / Crease & & & \\
& Other than skin fold & & 3 & 3 \\
Edema & Pitting & & & \\
Bleeding & Induced by trauma/abrasion & & 4 & \\
Ulceration & Spontaneous & 10 & & \\
Necrosis & & & & \\
\hline
\end{tabular}

CSSP: The modified 10-point Catterall skin scoring profile; RTOG: the Radiation Therapy Oncology Group; CTCAE: the Common Terminology Criteria for Adverse Events.

were delineated under the recommendations of the European Society for Radiotherapy and Oncology consensus guidelines (30). All patients underwent a standardized adjuvant RT protocol of 50 Gy to the whole breast delivered by a pair of wedged tangential fields (6-MV photon beams) in daily fractions of 2 Gy 5 days per week, followed by a $10 \mathrm{~Gy}$ electron boost to the tumor bed in daily fractions of $2 \mathrm{~Gy}$.

Study procedure and measurements. Participants were instructed to apply the allocated agent to the designated treatment site at least twice daily, starting on the first day of RT and for 4 weeks after completion of RT. All patients were advised to comply with the institutional skin care guideline: wear soft, loose cotton clothes and bras; avoid using soap and water; avoid sunlight exposure to the treatment area; and avoid using topical agents other than the allocated agents. If the patients allocated to the moisturizer group developed moist desquamation within the RT field, they were asked to discontinue applying X-derm ${ }^{\circledR}$ cream and to initiate wound dressings as per usual institutional practice. Because StrataXRT ${ }^{\circledR}$ can be used as a film-forming wound dressing for moist desquamation, the patients randomized to the silicone group were instructed to continue with the study protocol regardless of developing moist desquamation around the irradiated area.

The severity of RD was graded using physiological skin parameters, clinician-assessed visual rating scales, and patientreported symptoms. Changes in these parameters from baseline to 4 weeks post-RT were evaluated every two weeks (a total of approximately 10 weeks).

Physiological skin parameters. Skin toxicities induced by irradiation were assessed objectively using physiological skin parameters, including the EI, MI, and TEWL. These three physiological skin parameters were measured at the surface of the skin of the upper outer quadrant of the breast, at the midpoint between the axilla and nipple. This point was marked to ensure a consistent measurement location during the study period. Two experienced dermatologists performed measurements blinded to the arm allocation as well as to the results of other scoring criteria. The EI and MI were measured using a reflectance spectrophotometer (Mexameter ${ }^{\circledR}$ MX 18, Courage+Khazaka electronic $\mathrm{GmbH}$, Cologne, Germany) and were expressed as arbitrary Mexameter ${ }^{\circledR}$ units on a scale from 0 to 999 (11). The TEWL was measured using the Tewameter ${ }^{\circledR}$ TM 300 (Courage+Khazaka electronic GmbH, Cologne, Germany).

Visual rating scales and patient-reported symptoms. One radiation oncology nurse independently assessed the grade of RD using three visual rating scales, including the CSSP, RTOG, and CTCAE criteria, and the descriptions for each scale are summarized in Table I. Meanwhile, the patient-reported symptoms of dryness, itchiness, burning sensation, and pain in the treatment area were assessed using a 5-point scale questionnaire.

Statistical analysis. Statistical analysis was performed using $\mathrm{R}$ Statistical Software (version 3.6.1; R Foundation for Statistical Computing, Vienna, Austria). The student's t-test for continuous variables and Chi-square test for categorical variables were used to compare the differences in the data between groups according to their demographics and baseline variables. Continuous variables are reported as mean \pm standard deviation. Between-group differences in the changes of outcome variables were analysed using two-way repeated measures analysis of variance (ANOVA) with GreenhouseGeisser correction, which were expressed as F-statistic and $p$-value. We also conducted Bonferroni's correction to account for multiple testing (a total of six tests, therefore the $\alpha$ level was adjusted to $0.05 / 6=0.0083$ ). The correlations among the different physiological skin parameters and changes in clinician-assessed scoring criteria and patient-reported symptoms following RT were determined using Spearman's correlation test. A correlation coefficient, $\mathrm{Q}>0.6$ was considered to indicate a strong correlation, and $\varrho=0.3-0.6$ a moderate correlation. All the statistical tests were two-tailed and a significance level of 0.05 was accepted.

\section{Results}

A total of 56 patients were randomized during a 21-month recruitment period. Seven patients did not complete any of the post-RT assessments. Among them, five patients did not attend the 2 weeks post-RT assessment and two patients were lost to the final assessment. Thus, data for 49 patients (21 patients in the silicone group and 28 in the moisturizer group) were included in the analysis (Figure 1). The two groups were well-balanced in terms of clinical characteristics, except human epidermal growth factor receptor 2 (HER2) status and the use of trastuzumab (Table II). No differences in the results of baseline assessments were found between the groups.

Physiological skin parameters. The EI, measured using the Mexameter ${ }^{\circledR}$ MX 18, followed the same trajectory in both groups with a peak at the completion of RT (Figure 2A). There was a significant difference $(p=0.001)$ between groups when comparing the highest EI during the study period, with a 
Table II. Clinical characteristics of patients.

\begin{tabular}{|c|c|c|c|}
\hline Characteristics & $\begin{array}{c}\text { Silicone }^{\mathrm{a}} \\
(\mathrm{N}=21)\end{array}$ & $\begin{array}{l}\text { Moisturizer }{ }^{\mathrm{b}} \\
\quad(\mathrm{N}=28)\end{array}$ & $p$-Value \\
\hline Median age (range) & 46 years $(40-56)$ & 49 years $(29-60)$ & 0.135 \\
\hline BMI $\left(\mathrm{kg} / \mathrm{m}^{2}\right)$ & & & 0.587 \\
\hline$<25$ & $15(71.4 \%)$ & $23(82.1 \%)$ & \\
\hline $25-29$ & $6(28.6 \%)$ & $5(17.9 \%)$ & \\
\hline$\geq 30$ & 0 & 0 & \\
\hline Breast volume $\left(\mathrm{cm}^{3}\right)$ & $650.7 \pm 204.7$ & $559.5 \pm 242.9$ & 0.171 \\
\hline Boost volume $\left(\mathrm{cm}^{3}\right)$ & $56.2 \pm 29.8$ & $50.0 \pm 25.4$ & 0.437 \\
\hline Hypertension & & & 0.216 \\
\hline No & $20(95.2 \%)$ & $22(78.6 \%)$ & \\
\hline Yes & $1(4.8 \%)$ & $6(21.4 \%)$ & \\
\hline Diabetes mellitus & & & 1.000 \\
\hline No & $21(100.0 \%)$ & $27(96.4 \%)$ & \\
\hline Yes & 0 & $1(3.6 \%)$ & \\
\hline Laterality & & & 0.650 \\
\hline Left & $12(57.1 \%)$ & $13(46.4 \%)$ & \\
\hline Right & $9(42.9 \%)$ & $15(53.6 \%)$ & \\
\hline Breast cancer stage & & & 0.173 \\
\hline 0 & $1(4.8 \%)$ & $6(21.4 \%)$ & \\
\hline I & $11(52.4 \%)$ & $15(53.6 \%)$ & \\
\hline II & $9(42.9 \%)$ & $7(25.0 \%)$ & \\
\hline Estrogen receptor & & & 0.790 \\
\hline Negative & $3(14.3 \%)$ & $6(21.4 \%)$ & \\
\hline Positive & $18(85.7 \%)$ & $22(78.6 \%)$ & \\
\hline Progesterone receptor & & & 0.692 \\
\hline Negative & $8(38.1 \%)$ & $8(28.6 \%)$ & \\
\hline Positive & $13(61.9 \%)$ & $20(71.4 \%)$ & \\
\hline HER2 status & & & $0.021^{*}$ \\
\hline Negative & $13(61.9 \%)$ & $26(92.9 \%)$ & \\
\hline Positive & $8(38.1 \%)$ & $2(7.1 \%)$ & \\
\hline Chemotherapy & & & 0.269 \\
\hline No & $7(33.3 \%)$ & $18(64.3 \%)$ & \\
\hline CMF & $4(19.0 \%)$ & $3(10.7 \%)$ & \\
\hline AC-T & $6(28.6 \%)$ & $5(17.9 \%)$ & \\
\hline $\mathrm{AC}$ & $1(4.8 \%)$ & $1(3.6 \%)$ & \\
\hline $\mathrm{TC}$ & $3(14.3 \%)$ & $1(3.6 \%)$ & \\
\hline Hormone therapy & & & 1.000 \\
\hline No & $3(14.3 \%)$ & $5(17.9 \%)$ & \\
\hline Yes & $18(85.7 \%)$ & $23(82.1 \%)$ & \\
\hline Trastuzumab & & & $0.010 *$ \\
\hline No & $15(71.4 \%)$ & $28(100.0 \%)$ & \\
\hline Yes & $6(28.6 \%)$ & 0 & \\
\hline
\end{tabular}

BMI: Body mass index; HER2: human epidermal growth factor receptor 2; CMF: cyclophosphamide methotrexate and fluorouracil; AC-T: doxorubicin and cyclophosphamide followed by paclitaxel; AC: doxorubicin and cyclophosphamide; TC: taxotere and cyclophosphamide.

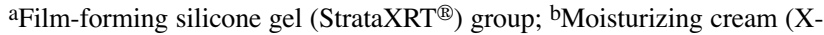
derm ${ }^{\circledR}$ ) group; ${ }^{*} p<0.05$.

higher EI in the moisturizer group than in the silicone group (Table III). This difference was verified by two-way repeated measures ANOVA, which showed significance ( $\mathrm{F}=3.609$, $p=0.008)$ in the interaction between time and group for the EI measurement (Figure 2A and Table IV). The post hoc analysis also demonstrated that the higher EI in the moisturizer group
Table III. Highest values of studied parameters during the study period.

\begin{tabular}{cccc}
\hline Parameter & Silicone & Moisturizer & $p$-Value \\
$(\mathrm{N}=21)$ & $(\mathrm{N}=28)$ &
\end{tabular}

\begin{tabular}{|c|c|c|c|}
\hline \multicolumn{4}{|l|}{$\begin{array}{l}\text { Physiological skin } \\
\text { parameters }\end{array}$} \\
\hline Erythema index ${ }^{c}$ & $453.4 \pm 61.6$ & $531.7 \pm 82.2$ & $0.001^{* *}$ \\
\hline Melanin index ${ }^{c}$ & $241.8 \pm 83.8$ & $314.0 \pm 85.5$ & $0.005^{* *}$ \\
\hline TEWL $\left(\mathrm{g} / \mathrm{h} / \mathrm{m}^{2}\right)$ & $15.3 \pm 10.2$ & $18.4 \pm 13.1$ & 0.374 \\
\hline \multicolumn{4}{|l|}{ Visual rating scales } \\
\hline CSSP & & & 0.650 \\
\hline 3 & $2(9.5 \%)$ & $2(7.1 \%)$ & \\
\hline 4 & $0(0.0 \%)$ & $2(7.1 \%)$ & \\
\hline 5 & $18(85.7 \%)$ & $23(82.1 \%)$ & \\
\hline 6 & $1(4.8 \%)$ & $1(3.6 \%)$ & \\
\hline RTOG & & & - \\
\hline 1 & $21(100.0 \%)$ & $28(100.0 \%)$ & \\
\hline CTCAE & & & - \\
\hline 1 & $21(100.0 \%)$ & $28(100.0 \%)$ & \\
\hline \multicolumn{4}{|c|}{ Patient-reported symptoms } \\
\hline Dryness & & & 0.188 \\
\hline 0 & $6(28.6 \%)$ & $11(39.3 \%)$ & \\
\hline 1 & 0 & $6(21.4 \%)$ & \\
\hline 2 & $5(23.8 \%)$ & $3(10.7 \%)$ & \\
\hline 3 & $7(33.3 \%)$ & $5(17.9 \%)$ & \\
\hline 4 & $2(9.5 \%)$ & $2(7.1 \%)$ & \\
\hline 5 & $1(4.8 \%)$ & $1(3.6 \%)$ & \\
\hline Itching & & & 0.463 \\
\hline 0 & $5(23.8 \%)$ & $5(17.9 \%)$ & \\
\hline 1 & $5(23.8 \%)$ & $5(17.9 \%)$ & \\
\hline 2 & $2(9.5 \%)$ & $9(32.1 \%)$ & \\
\hline 3 & $7(33.3 \%)$ & $6(21.4 \%)$ & \\
\hline 4 & $2(9.5 \%)$ & $2(7.1 \%)$ & \\
\hline 5 & 0 & $1(3.6 \%)$ & \\
\hline Burning & & & 0.094 \\
\hline 0 & $5(23.8 \%)$ & $9(32.1 \%)$ & \\
\hline 1 & $2(9.5 \%)$ & $7(25 \%)$ & \\
\hline 2 & $9(42.9 \%)$ & $5(17.9 \%)$ & \\
\hline 3 & $4(19 \%)$ & $3(10.7 \%)$ & \\
\hline 4 & 0 & $4(14.3 \%)$ & \\
\hline 5 & $1(4.8 \%)$ & 0 & \\
\hline Pain & & & 0.262 \\
\hline 0 & $4(19.0 \%)$ & $5(17.9 \%)$ & \\
\hline 1 & $3(14.3 \%)$ & $5(17.9 \%)$ & \\
\hline 2 & $2(9.5 \%)$ & $8(28.6 \%)$ & \\
\hline 3 & $7(33.3 \%)$ & $3(10.7 \%)$ & \\
\hline 4 & $5(23.8 \%)$ & $7(25.0 \%)$ & \\
\hline 5 & 0 & 0 & \\
\hline
\end{tabular}

TEWL: Trans-epidermal water loss. ${ }^{\text {aFilm-forming silicone gel }}$ (StrataXRT $\left.{ }^{\circledR}\right)$ group; b Moisturizing cream $\left(\mathrm{X}\right.$-derm $\left.{ }^{\circledR}\right)$ group; ${ }^{\mathrm{c}}$ Arbitrary Mexameter $^{\circledR}$ units (on a scale from 0 to 999 ); $*^{*} p<0.01$.

at 6 weeks (completion of RT) was statistically highly significant $(p<0.001$, Table IV).

The MI in both groups increased from baseline to 2 weeks after completion of RT and then decreased (Figure 2B). The highest MI during the study period in the moisturizer group was significantly higher than that in the silicone group 


\section{Physiological skin parameters}
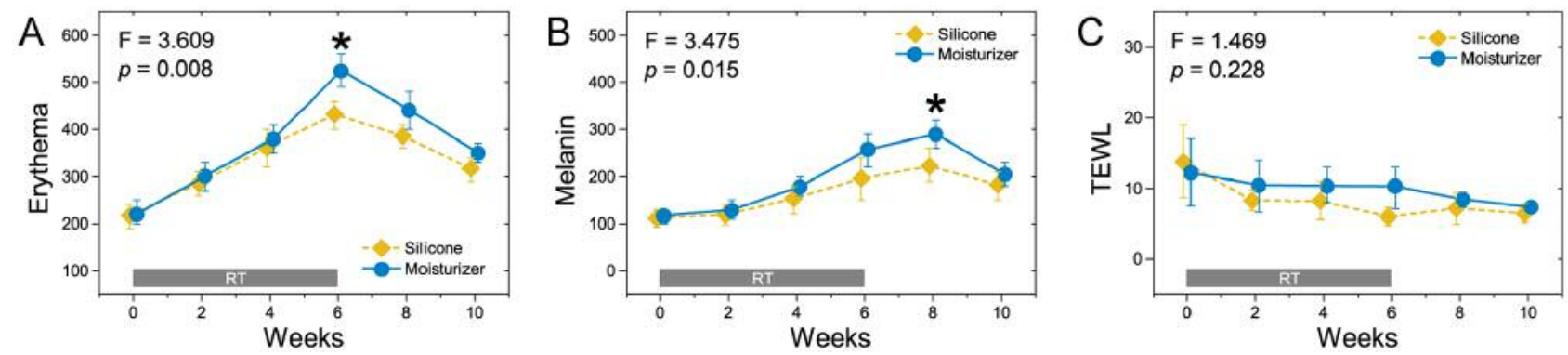

\section{Visual rating scales}
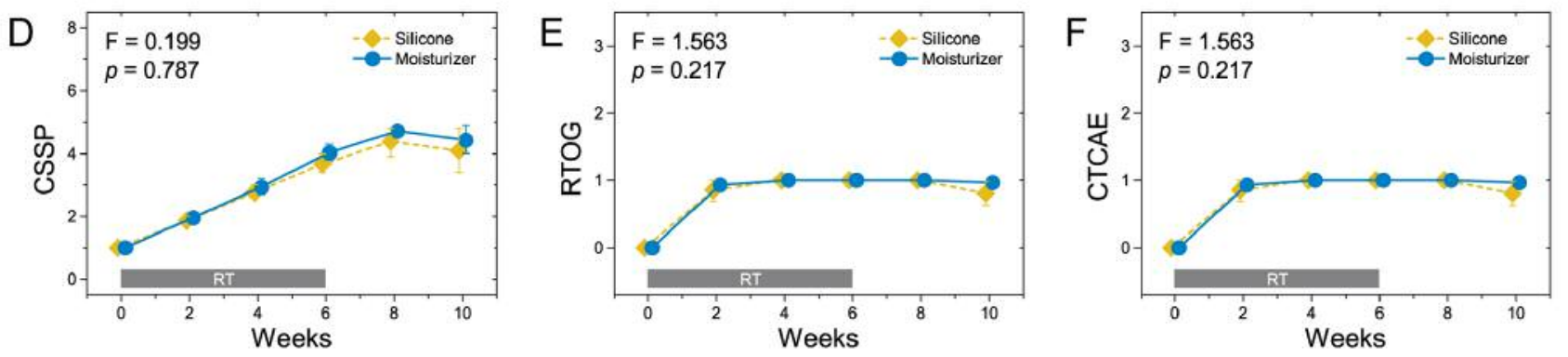

\section{Patient-reported symptoms}
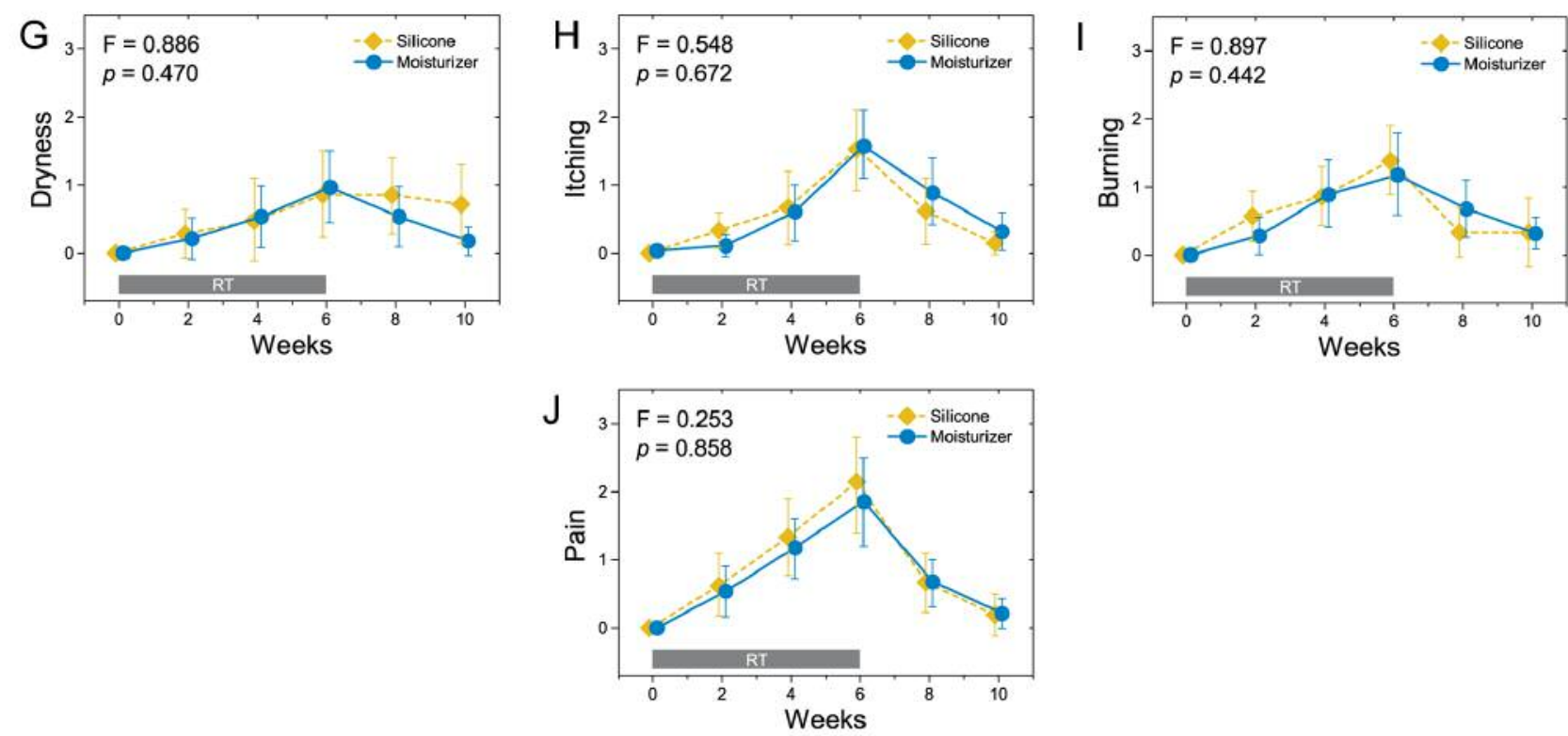

Figure 2. Changes in the severity of radiation dermatitis in the Silicone group (StrataXRT $\left.{ }^{\circledR}\right)$ and the Moisturizer group $\left(X\right.$-derm $\left.{ }^{\circledR}\right)$. Data are expressed as means (95\% confidence intervals). Two-way repeated measures ANOVA with Greenhouse-Geisser correction followed by Bonferroni's post hoc test were used to verify the statistical significance of interaction between time and group factors, which were expressed as $F$-statistic and p-value. A: Erythema index (arbitrary Mexameter ${ }^{\circledR}$ units on a scale from 0 to 999). B: Melanin index (arbitrary Mexameter ${ }^{\circledR}$ units on a scale from 0 to 999). C: Trans-epidermal water loss (TEWL, $\left.\mathrm{g} / \mathrm{h} / \mathrm{m}^{2}\right)$. D: Modified 10-point Catterall skin scoring profile. E: Radiation Therapy Oncology Group (RTOG) criteria. F: Common Terminology Criteria for Adverse Events. G: Dryness. H: Itching. I: Burning sensation. J: Pain. *p<0.0083, Bonferroni's post hoc test for multiple comparisons. 
Table IV. Changes in physiological skin parameters and the results of two-way repeated-measures ANOVA with Greenhouse-Geisser correction for the interaction between time and group factors.

\begin{tabular}{|c|c|c|c|c|}
\hline Parameter & Silicone $^{\mathrm{a}}$ & Moisturizer ${ }^{b}$ & F-Statistic & $p$-Value \\
\hline Erythema Index ${ }^{c}$ & & & 3.609 & 0.008 \\
\hline Baseline & $217.2 \pm 60.5$ & $220.5 \pm 63.4$ & & \\
\hline 2 weeks & $285.5 \pm 52.3$ & $301.1 \pm 81.8$ & & \\
\hline 4 weeks & $361.0 \pm 89.2$ & $380.1 \pm 70.2$ & & \\
\hline 6 weeks & $432.9 \pm 64.6$ & $525.0 \pm 80.7$ & & $\left(<0.001^{*}\right)$ \\
\hline 8 weeks & $386.8 \pm 58.9$ & $441.6 \pm 104.4$ & & \\
\hline 10 weeks & $316.7 \pm 54.0$ & $350.6 \pm 56.6$ & & \\
\hline Melanin Index ${ }^{c}$ & & & 3.475 & 0.015 \\
\hline Baseline & $111.6 \pm 40.9$ & $117.4 \pm 41.0$ & & \\
\hline 2 weeks & $118.5 \pm 47.3$ & $129.1 \pm 48.0$ & & \\
\hline 4 weeks & $153.3 \pm 65.4$ & $179.0 \pm 60.9$ & & \\
\hline 6 weeks & $196.4 \pm 93.6$ & $257.5 \pm 85.7$ & & \\
\hline 8 weeks & $221.9 \pm 78.0$ & $290.1 \pm 85.9$ & & $\left(0.006^{*}\right)$ \\
\hline 10 weeks & $183.0 \pm 65.6$ & $204.3 \pm 66.9$ & & \\
\hline Trans-Epidermal Water Loss $\left(\mathrm{g} / \mathrm{h} / \mathrm{m}^{2}\right)$ & & & 1.469 & 0.228 \\
\hline Baseline & $14.8 \pm 11.6$ & $12.4 \pm 12.3$ & & \\
\hline 2 weeks & $8.2 \pm 3.1$ & $10.7 \pm 10.0$ & & \\
\hline 4 weeks & $10.1 \pm 6.2$ & $11.2 \pm 7.3$ & & \\
\hline 6 weeks & $6.4 \pm 2.9$ & $11.3 \pm 8.8$ & & \\
\hline 8 weeks & $7.4 \pm 5.3$ & $9.0 \pm 3.9$ & & \\
\hline 10 weeks & $6.7 \pm 3.1$ & $7.9 \pm 2.3$ & & \\
\hline
\end{tabular}

aFilm-forming silicone gel (StrataXRT®) group; b Moisturizing cream (X-derm ${ }^{\circledR}$ ) group; ${ }^{\mathrm{c}}$ Arbitrary Mexameter ${ }^{\circledR}$ units (on a scale from 0 to 999 ); ${ }^{*} p<0.0083$, Bonferroni's post hoc test for multiple comparisons implemented, and an $\alpha$ value less than 0.0083 was required for statistical significance.

$(p=0.005$, Table III). A significant interaction between time and group in the changes in the $\mathrm{MI}(\mathrm{F}=3.475, p=0.015)$ was observed based on two-way repeated measures ANOVA (Figure 2B and Table IV). The post hoc analysis demonstrated a significantly higher MI in the moisturizer group compared with that in the silicone group 2 weeks after completion of RT $(p<0.001$, Table IV).

TEWL decreased continuously during the study period (Figure 2C). There were no significant differences between the groups in the TEWL measurements (Table IV).

Visual rating scales. Most patients developed mild RD, and no patient experienced edema or moist desquamation. When the CSSP was used, the majority of patients were scored as grade 5 (Table III). All patients were scored as grade 1 using the RTOG and CTCAE criteria (Table III). There were no between-group differences in the changes in clinicianassessed visual rating scales during the study period (Figure 2D-F).

Patient-reported symptoms. The progression of patientreported symptoms evaluated using the 5-point Likert scale showed a similar trajectory (Figure 2G-J). These scores representing dryness, itchiness, burning sensation, and pain gradually increased during RT, reached a peak at the end of RT, and decreased thereafter. No statistically significant differences between the groups were observed in the patientreported symptoms.

Correlation of physiological skin parameters with visual rating scales and patient-reported symptoms. When analysing the correlations among the three different physiological skin parameters, we found a strong correlation between EI and MI (Table V). TEWL showed no significant correlation with other physiological skin parameters.

Regarding the clinician-assessed visual rating scales, CSSP revealed strong correlations with EI and MI (Table V). The RTOG and CTCAE criteria correlated moderately with EI and MI. TEWL showed no significant correlation with visual rating scales. There were no strong correlations between physiological skin parameters and patient-reported symptoms.

\section{Discussion}

In the present study, we prospectively evaluated the clinical efficacy of a film-forming silicone gel on the prevention of $\mathrm{RD}$ in comparison with a moisturizing cream. Our results demonstrated significant decreases in EI and MI in the patients allocated to the StrataXRT group. To our knowledge, this is the first randomized trial demonstrating the superiority of a film-forming silicone gel in reducing 
Table V. Correlations among physiological skin parameters, clinicianassessed visual rating scales, and patient-reported symptoms.

\begin{tabular}{lcll}
\hline Parameter & EI & MI & TEWL \\
\hline $\begin{array}{l}\text { Physiological skin parameters } \\
\text { EI }\end{array}$ & & & \\
MI & & $0.63^{* * * *}$ & $-0.14^{*}$ \\
$\quad$ TEWL $\left(\mathrm{g} / \mathrm{h} / \mathrm{m}^{2}\right)$ & $0.63^{* * *}$ & & -0.10 \\
Visual Rating Scales & $-0.14^{*}$ & -0.10 & \\
CSSP & & & \\
RTOG & $0.61^{* * *}$ & $0.56^{* * *}$ & $-0.20^{* * *}$ \\
CTCAE & $0.56^{* * *}$ & $0.38^{* * *}$ & $-0.22^{* * *}$ \\
Patient-reported symptoms & $0.56^{* * *}$ & $0.38^{* * *}$ & $-0.22^{* * *}$ \\
$\quad$ & & & \\
Dryness & $0.18^{* *}$ & 0.07 & 0.05 \\
Itching & $0.45^{* * *}$ & $0.24^{* * *}$ & -0.07 \\
Burning sensation & $0.32^{* * *}$ & 0.05 & -0.04 \\
Pain & $0.38^{* * *}$ & $0.15^{*}$ & 0.05 \\
\hline
\end{tabular}

EI: Erythema index; MI: melanin index; TEWL: trans-epidermal water loss; CSSP: the modified 10-point Catterall skin scoring profile; RTOG: the Radiation Therapy Oncology Group scale; CTCAE: the Common Terminology Criteria for Adverse Events scale. ${ }^{\text {aArbitrary Mexameter }}{ }^{\circledR}$ units (on a scale from 0 to 999 ); ${ }^{*} p<0.05,{ }^{* *} p<0.01,{ }^{* * *} p<0.001$.

RD during breast RT based on objectively measured physiological skin parameters.

Despite decades of investigations, no clear evidence of the superiority of any single topical agent in the prevention of RD has emerged $(10,17-22,24)$. Although steroids have been proven to be potent topical agents in reducing RD (31, 32 ), the use of steroids requires a doctor's prescription and is not free of criticism. Regardless of the type of topical agents, previous studies have evaluated the severity of RD using clinician-assessed scales or patient-reported symptom scores. Consistent with previous studies, our study also demonstrated that there were no differences between study groups in the severity of RD assessed by three visual rating scales and four patient-reported symptoms. These subjective scales have many drawbacks, which can compromise the accuracy and reliability of detecting radiation-induced skin changes. Many studies have pointed out such drawbacks, including inter- and intra-observer variations and the lack of objectivity $(14,33)$.

In this context, the need for more objective measurements of the severity of RD have emerged. Advances in technology have allowed many physiological skin parameters to be measured non-invasively (11-15). As an example, LDF has been proposed to assess RD by measuring cutaneous blood flow and has shown promising results in detecting radiationinduced changes even before the clinical manifestation of RD became evident, such as grade 0 dermatitis (15). In our study cohort, no patient experienced moist desquamation, and most patients developed CSSP grade 5 radiation dermatitis, which corresponds to grade 1 on the RTOG and
CTCAE criteria (Table III). Similar to the results of the LDF measurement, we found that the quantitatively measured EI and MI can distinguish the difference in radiation-induced changes between study groups, even in patients experiencing very low-grade RD.

Previous studies have shown that impaired epidermal function induced by RT leads to an increase in TEWL (34). As shown in Figure 2C, TEWL values continued to decline throughout the study period. This was an unexpected finding and may be due to the fact that various topical agents can alter the results of TEWL measurement regardless of skin condition $(35,36)$. The continuous use of topical agents in accordance with study instructions may maintain a moist skin environment, leading to a decrease in TEWL.

Although numerous physiological skin parameters have been introduced to quantify radiation-induced skin changes, various subjective criteria such as clinician- and patientassessed scales are widely used as the most common standard tools to evaluate the severity of RD. For this reason, there is a need to identify the concordance among these different parameters. Previously, various objective parameters including pigmentation, hydration, and $\mathrm{pH}$ of the skin, were tested in patients receiving breast RT, and only cutaneous blood flow measured by LDF has shown a strong correlation with the RTOG and CTCAE criteria $(14,15)$. Skin pigmentation have also been found to be moderately correlated with clinician-assessed scales. The results of the present study demonstrated that objectively measured EI and MI showed a moderate correlation with clinician-assessed visual rating scales, confirming previous results. On the other hand, we found that EI strongly correlated with the CSSP, which was considered a more sophisticated version with the 10-point grading scale.

$\mathrm{RD}$ is a series of processes that undergo development, deterioration, and recovery. To evaluate these processes, we should repeatedly measure the outcome variables associated with skin changes over time. Such data repeatedly measured from the same individuals are referred to as longitudinal data (37). Appropriate analysis of the longitudinal data requires specific statistical attention. Nevertheless, most studies have used the maximum peak scores or the number of patients experiencing severe RD as an endpoint of statistical analyses $(10,19,22,24)$. Survival analysis dealing with the time of diagnosis of a certain grade of skin toxicity have also been conducted (29). However, these summary statistic approaches, in which all individual measurements are condensed in a single parameter, can lose a substantial amount of information (38), such as changes in the recovery period. In the present study, repeatedly measured skin parameters were analysed by using two-way repeated measures ANOVA $(37,39)$. Using this technique, analysis of the interactions between time effects, group effects, and time and group factors enabled comprehensive interpretation of 
longitudinal data, and finally, identification of differences between groups in reducing RD.

In our study design, the data measuring radiation-induced skin changes were collected at two-week intervals during RT. Post-RT assessments were also conducted every two weeks to account for our routine follow-up interval of four weeks. In general, the severity of $\mathrm{RD}$ reached its maximum value around 5-7 weeks from baseline. Previous studies have revealed that the severity of RD shows a peak one week after completion of RT (10). We have no data collected at that period, and the assessment schedule of the two-week interval may lead to a decrease in the maximum value of the parameters examined in this study.

The present study has limitations. First, this randomized controlled trial with small patient numbers was unblinded, and some bias cannot be excluded. However, physiological skin parameters and clinician-assessed symptoms were assessed independently by each researcher blinded to the results of other scoring systems. Participants also completed the questionnaire without any information about the assessment results. Thus, this limitation would have minimal impact on the outcome measurements of this trial. Second, physiological skin parameters were measured only using the EI, MI and TEWL. Although numerous techniques have been used for the objective measurement of quantitative skin parameters, there is no gold standard for diagnosing and grading the severity of RD. Therefore, various skin parameters should be evaluated in terms of clinical correlation and concordance between the techniques. Finally, no patients experienced moist desquamation, unlike the incidences in previously published reports. This study should be reproduced to confirm the efficacy of StrataXRT in more severe cases of RD.

\section{Conclusion}

This pilot study showed that topical use of a film-forming silicone gel (StrataXRT) can reduce RD with respect to objectively measured physiological skin parameters. The outcomes of this trial support the feasibility of conducting a larger randomized controlled trial.

\section{Conflicts of Interest}

The Authors declare that they have no competing interests regarding this study.

\section{Authors' Contributions}

SA provided ideas. SA and KS drafted the manuscript. SA, KS, JSK, and SKL conducted patient examinations and participated in data collection. SA, KS, and YEC collected patient data and performed the statistical analysis. KS, HJK, YEC, and YKL were responsible for patient treatment and radiation therapy planning. JYR, HJK, and YEC supervised the analysis and interpretation of the data and reviewed the manuscript. All Authors read and approved the final manuscript.

\section{Acknowledgements}

This work was supported by the Gachon University Gil Medical Center (Grant number: 2017-15).

\section{References}

1 Mikeljevic JS, Haward R, Johnston C, Crellin A, Dodwell D, Jones A, Pisani $\mathrm{P}$ and Forman D: Trends in postoperative radiotherapy delay and the effect on survival in breast cancer patients treated with conservation surgery. Br J Cancer 90: 13431348, 2004. PMID: 15054452. DOI: 10.1038/sj.bjc.6601693

2 Gosselin TK, Schneider SM, Plambeck MA and Rowe K: A prospective randomized, placebo-controlled skin care study in women diagnosed with breast cancer undergoing radiation therapy. Oncol Nurs Forum 37: 619-626, 2010. PMID: 20797953. DOI: $10.1188 / 10.0 n f .619-626$

3 Kole AJ, Kole L and Moran MS: Acute radiation dermatitis in breast cancer patients: challenges and solutions. Breast Cancer (Dove Med Press) 9: 313-323, 2017. PMID: 28503074. DOI: $10.2147 /$ bctt.S109763

4 Hegedus F, Mathew LM and Schwartz RA: Radiation dermatitis: an overview. Int J Dermatol 56: 909-914, 2017. PMID: 27496623. DOI: $10.1111 / \mathrm{ijd} .13371$

5 Ryan JL: Ionizing radiation: the good, the bad, and the ugly. J Invest Dermatol 132: 985-993, 2012. PMID: 22217743. DOI: 10.1038/jid.2011.411

6 Arenas M, Sabater S, Gascon M, Henriquez I, Bueno MJ, Rius A, Rovirosa A, Gomez D, Lafuerza A, Biete A and Colomer J: Quality assurance in radiotherapy: analysis of the causes of not starting or early radiotherapy withdrawal. Radiat Oncol 9: 260, 2014. PMID: 25472662. DOI: 10.1186/s13014-014-0260-0

7 National Cancer Institute: Common Terminology Criteria for Adverse Events (CTCAE) v4.03 (2010). Available at: https://ctep.cancer.gov/protocolDevelopment/electronic_applicati ons/ctc.htm

8 Cox JD, Stetz J and Pajak TF: Toxicity criteria of the Radiation Therapy Oncology Group (RTOG) and the European Organization for Research and Treatment of Cancer (EORTC). Int J Radiat Oncol Biol Phys 31: 1341-1346, 1995. PMID: 7713792. DOI: 10.1016/0360-3016(95)00060-c

9 Catterall M, Rogers C, Thomlinson RH and Field SB: An investigation into the clinical effects of fast neutrons. Methods and early observations. Br J Radiol 44: 603-611, 1971. PMID: 4997649. DOI: 10.1259/0007-1285-44-524-603

10 Hoopfer D, Holloway C, Gabos Z, Alidrisi M, Chafe S, Krause B, Lees A, Mehta N, Tankel K, Strickland F, Hanson J, King C, Ghosh S and Severin D: Three-arm randomized phase III trial: Quality aloe and placebo cream versus powder as skin treatment during breast cancer radiation therapy. Clin Breast Cancer 15: 181-190.e181-184, 2015. PMID: 25619686. DOI: 10.1016/ j.clbc.2014.12.006

11 Wright CY, Karsten AE, Wilkes M, Singh A, du Plessis J, Albers PN and Karsten PA: Diffuse reflectance spectroscopy versus mexameter((R)) MX18 measurements of melanin and erythema in an african population. Photochem Photobiol 92: 632-636, 2016. PMID: 27276188. DOI: 10.1111/php.12607 
12 Chan MWM, Shek SY, Yeung CK and Chan HH: A prospective study in the treatment of lentigines in Asian skin using $532 \mathrm{~nm}$ picosecond Nd:YAG laser. Lasers Surg Med, 2019. PMID: 31115070. DOI: $10.1002 / \mathrm{lsm} .23103$

13 Pinnagoda J, Tupker RA, Agner T and Serup J: Guidelines for transepidermal water loss (TEWL) measurement. A report from the Standardization Group of the European Society of Contact Dermatitis. Contact Dermatitis 22: 164-178, 1990. PMID: 2335090. DOI: 10.1111/j.1600-0536.1990.tb01553.x

14 Huang CJ, Hou MF, Luo KH, Wei SY, Huang MY, Su SJ, Kuo HY, Yuan SS, Chen GS, Hu SC and Chuang HY: RTOG, CTCAE and WHO criteria for acute radiation dermatitis correlate with cutaneous blood flow measurements. Breast 24: 230-236, 2015. PMID: 25777626. DOI: 10.1016/j.breast. 2015.01 .008

15 Gonzalez Sanchis A, Brualla Gonzalez L, Sanchez Carazo JL, Gordo Partearroyo JC, Esteve Martinez A, Vicedo Gonzalez A and Lopez Torrecilla JL: Evaluation of acute skin toxicity in breast radiotherapy with a new quantitative approach. Radiother Oncol 122: 54-59, 2017. PMID: 27825796. DOI: 10.1016/ j.radonc.2016.09.019

16 Porock D and Kristjanson L: Skin reactions during radiotherapy for breast cancer: the use and impact of topical agents and dressings. Eur J Cancer Care (Engl) 8: 143-153, 1999. PMID: 10763645.

17 Haruna F, Lipsett A and Marignol L: Topical management of acute radiation dermatitis in breast cancer patients: a systematic review and meta-analysis. Anticancer Res 37: 5343-5353, 2017. PMID: 28982842. DOI: 10.21873/anticanres.11960

18 Salvo N, Barnes E, van Draanen J, Stacey E, Mitera G, Breen D, Giotis A, Czarnota G, Pang J and De Angelis C: Prophylaxis and management of acute radiation-induced skin reactions: a systematic review of the literature. Curr Oncol 17: 94-112, 2010. PMID: 20697521. DOI: 10.3747/co.v17i4.493

19 Pinnix C, Perkins GH, Strom EA, Tereffe W, Woodward W, Oh JL, Arriaga L, Munsell MF, Kelly P, Hoffman KE, Smith BD, Buchholz TA and Yu TK: Topical hyaluronic acid $v s$. standard of care for the prevention of radiation dermatitis after adjuvant radiotherapy for breast cancer: single-blind randomized phase III clinical trial. Int J Radiat Oncol Biol Phys 83: 1089-1094, 2012. PMID: 22172912. DOI: 10.1016/j.ijrobp.2011.09.021

20 Wells M, Macmillan M, Raab G, MacBride S, Bell N, MacKinnon K, MacDougall H, Samuel L and Munro A: Does aqueous or sucralfate cream affect the severity of erythematous radiation skin reactions? A randomised controlled trial. Radiother Oncol 73: 153-162, 2004. PMID: 15542162. DOI: 10.1016/j.radonc.2004.07.032

21 Cleary JF, Anderson BM, Eickhoff JC, Khuntia D and Fahl WE: Significant suppression of radiation dermatitis in breast cancer patients using a topically applied adrenergic vasoconstrictor. Radiat Oncol 12: 201, 2017. PMID: 29273054. DOI: 10.1186/s13014-017-0940-7

22 Graham PH, Plant N, Graham JL, Browne L, Borg M, Capp A, Delaney GP, Harvey J, Kenny L, Francis M and Zissiadis Y: A paired, double-blind, randomized comparison of a moisturizing durable barrier cream to $10 \%$ glycerine cream in the prophylactic management of postmastectomy irradiation skin care: trans Tasman Radiation Oncology Group (TROG) 04.01 Int J Radiat Oncol Biol Phys 86: 45-50, 2013. PMID: 23414763. DOI: 10.1016/j.ijrobp.2012.12.009
23 Diggelmann KV, Zytkovicz AE, Tuaine JM, Bennett NC, Kelly LE and Herst PM: Mepilex Lite dressings for the management of radiation-induced erythema: a systematic inpatient controlled clinical trial. Br J Radiol 83: 971-978, 2010. PMID: 20647511. DOI: $10.1259 / \mathrm{bjr} / 62011713$

24 Herst PM, Bennett NC, Sutherland AE, Peszynski RI, Paterson DB and Jasperse ML: Prophylactic use of Mepitel Film prevents radiation-induced moist desquamation in an intra-patient randomised controlled clinical trial of 78 breast cancer patients. Radiother Oncol 110: 137-143, 2014. PMID: 24486117. DOI: 10.1016/j.radonc.2014.01.005

25 Mustoe TA: Evolution of silicone therapy and mechanism of action in scar management. Aesthetic Plast Surg 32: 82-92, 2008. PMID: 17968615. DOI: 10.1007/s00266-007-9030-9

26 Sandhofer M and Schauer P: The safety, efficacy, and tolerability of a novel silicone gel dressing following dermatological surgery. Skinmed 10: S1-7, 2012. PMID: 23346665.

27 Quilis A, Martín J, Rodríguez C, Sánchez P and Luis Ribes J: Reducing radiation dermatitis during ongoing radiation therapy: an innovative film-forming wound dressing. J Radiat Oncol 7, 2018. DOI: $10.1007 / \mathrm{s} 13566-018-0356-5$

28 Arranz Villandiego I and Gonzalez Aramberri S: The use of an innovative film-forming wound dressing in the treatment of radiation dermatitis during concomitant chemotherapy. J Cancer Ther 09: 1048-1056, 2018. DOI: 10.4236/jct. 2018.912086

29 Chan RJ, Blades R, Jones L, Downer T-R, Peet SC, Button E, Wyld D, McPhail S, Doolan M and Yates P: A single-blind, randomised controlled trial of StrataXRT(R) - A silicone-based film-forming gel dressing for prophylaxis and management of radiation dermatitis in patients with head and neck cancer. Radiother Oncol 139: 72-78, 2019. PMID: 31445838. DOI: 10.1016/j.radonc.2019.07.014

30 Offersen BV, Boersma LJ, Kirkove C, Hol S, Aznar MC, Biete Sola A, Kirova YM, Pignol JP, Remouchamps V, Verhoeven K, Weltens C, Arenas M, Gabrys D, Kopek N, Krause M, Lundstedt D, Marinko T, Montero A, Yarnold J and Poortmans P: ESTRO consensus guideline on target volume delineation for elective radiation therapy of early stage breast cancer. Radiother Oncol 114: 3-10, 2015. PMID: 25630428. DOI: 10.1016/j.radonc.2014.11.030

31 Miller RC, Schwartz DJ, Sloan JA, Griffin PC, Deming RL, Anders JC, Stoffel TJ, Haselow RE, Schaefer PL, Bearden JD, 3rd, Atherton PJ, Loprinzi CL and Martenson JA: Mometasone furoate effect on acute skin toxicity in breast cancer patients receiving radiotherapy: a phase III double-blind, randomized trial from the North Central Cancer Treatment Group N06C4. Int J Radiat Oncol Biol Phys 79: 1460-1466, 2011. PMID: 20800381. DOI: 10.1016/j.ijrobp.2010.01.031

32 Ulff E, Maroti M, Serup J and Falkmer U: A potent steroid cream is superior to emollients in reducing acute radiation dermatitis in breast cancer patients treated with adjuvant radiotherapy. A randomised study of betamethasone versus two moisturizing creams. Radiother Oncol 108: 287-292, 2013. PMID: 23827771. DOI: 10.1016/j.radonc.2013.05.033

33 Mukesh M, Harris E, Jena R, Evans $\mathrm{P}$ and Coles C: Relationship between irradiated breast volume and late normal tissue complications: a systematic review. Radiother Oncol 104: 1-10, 2012. PMID: 22682540. DOI: $10.1016 /$ j.radonc .2012 .04 .025 
34 Hymes SR, Strom EA and Fife C: Radiation dermatitis: clinical presentation, pathophysiology, and treatment 2006. J Am Acad Dermatol 54: 28-46, 2006. PMID: 16384753. DOI: 10.1016/ j.jaad.2005.08.054

35 Dahnhardt-Pfeiffer S, Dahnhardt D, Buchner M, Walter K, Proksch E and Folster-Holst R: Comparison of effects of tacrolimus ointment and mometasone furoate cream on the epidermal barrier of patients with atopic dermatitis. J Dtsch Dermatol Ges 11: 437-443, 2013. PMID: 23551950. DOI: $10.1111 /$ ddg. 12074

36 Loden M: The increase in skin hydration after application of emollients with different amounts of lipids. Acta Derm Venereol 72: 327-330, 1992. PMID: 1361276.

37 Albert PS: Longitudinal data analysis (repeated measures) in clinical trials. Stat Med 18: 1707-1732, 1999. PMID: 10407239.
38 Schober $\mathrm{P}$ and Vetter TR: repeated measures designs and analysis of longitudinal data: if at first you do not succeed-try, try again. Anesth Analg 127: 569-575, 2018. PMID: 29905618. DOI: $10.1213 /$ ane. 0000000000003511

39 Park E, Cho $\mathrm{M}$ and Ki CS: Correct use of repeated measures analysis of variance. Korean J Lab Med 29: 1-9, 2009. PMID: 19262072. DOI: $10.3343 / \mathrm{kjlm} .2009 .29 .1 .1$

Received September 25, 2019

Revised October 9, 2019

Accepted October 10, 2019 